\title{
Burden of diabetes in Australia: life expectancy and disability-free life expectancy in adults with diabetes
}

\author{
Lili Huo $^{1,2}$ • Jonathan E. Shaw ${ }^{2,3}$ • Evelyn Wong ${ }^{2,3,4}$ • Jessica L. Harding ${ }^{2,3}$. \\ Anna Peeters ${ }^{2,3,4}$ - Dianna J. Magliano ${ }^{2,3}$
}

Received: 8 December 2015 / Accepted: 8 March 2016 / Published online: 14 April 2016

(C) Springer-Verlag Berlin Heidelberg 2016

\begin{abstract}
Aims/hypothesis The aim of this work was to estimate the life expectancy (LE) and disability-free life expectancy (DFLE) for adults with and without diabetes.

Methods The Chiang method and the adapted Sullivan method were used to estimate LE and DFLE by age and sex. Mortality data in 2011 were available from the National Diabetes Services Scheme for diabetes and from standard national mortality datasets for the general population. Data on prevalence of disability and severe or profound core activity limitation were derived from the 2012 Australian Survey of Disability, Ageing and Carers (SDAC). The definitions of disability used in the SDAC followed the International Classification of Functioning, Disability and Health. Data on diabetes prevalence were derived from the Australian Diabetes, Obesity and Lifestyle study.
\end{abstract}

Anna Peeters and Dianna J. Magliano are joint senior authors.

Electronic supplementary material The online version of this article (doi:10.1007/s00125-016-3948-x) contains peer-reviewed but unedited supplementary material, which is available to authorised users.

\section{Lili Huo}

Lili.Huo@bakeridi.edu.au

1 Department of Endocrinology, Beijing Jishuitan Hospital, No. 31, Xinjiekou East Street, Xicheng District, Beijing 100035, People's Republic of China

2 Department of Clinical Diabetes and Epidemiology, Baker IDI Heart and Diabetes Institute, Melbourne, VIC, Australia

3 Department of Epidemiology and Preventive Medicine, Monash University, Melbourne, VIC, Australia

4 School of Health and Social Development, Deakin University, Melbourne, VIC, Australia
Results The estimated LE and DFLE (with 95\% uncertainty interval [UI]) at age 50 years were $30.2(30.0,30.4)$ and 12.7 $(11.5,13.7)$ years, respectively, for men with diabetes, and the estimates were $33.9(33.6,34.1)$ and $13.1(12.3,13.9)$ years, respectively, for women with diabetes. The estimated loss of LE associated with diabetes at age 50 years was $3.2(3.0,3.4)$ years for men and $3.1(2.9,3.4)$ years for women, as compared with their counterparts without diabetes. The corresponding estimated loss of DFLE was $8.2(6.7,9.7)$ years for men and $9.1(7.9,10.4)$ years for women. Women with diabetes spent a greater number of absolute years and a greater proportion of their life with disability as compared with men with diabetes and women without diabetes. The gains in LE and DFLE across the whole population at age 50 years after hypothetically eliminating diagnosed diabetes were $0.6(0.5,0.6)$ years and $1.8(1.0,2.8)$ years.

Conclusions/interpretation In adults, diabetes results in a modest reduction in LE and a substantial reduction in DFLE. Efforts to identify the specific causes of disability and effective interventions are needed.

Keywords Diabetes $\cdot$ Disability $\cdot$ Disability-free life expectancy $\cdot$ Life expectancy

$\begin{array}{ll}\text { Abbreviations } \\ \text { ABS } & \text { Australian Bureau of Statistics } \\ \text { ADLs } & \text { Activities of daily living } \\ \text { AIHW } & \text { Australian Institute of Health and Welfare } \\ \text { AusDiab } & \text { Australian Diabetes, Obesity and Lifestyle } \\ \text { DALYs } & \text { Disability-adjusted life-years } \\ \text { DFLE } & \text { Disability-free life expectancy } \\ \text { HALE } & \text { Health-adjusted life expectancy } \\ \text { ICF } & \text { International Classification of Functioning, } \\ & \text { Disability and Health }\end{array}$

Abbreviations

ABS Australian Bureau of Statistics

ADLs Activities of daily living

AIHW Australian Institute of Health and Welfare

AusDiab Australian Diabetes, Obesity and Lifestyle

DALYs Disability-adjusted life-years

DFLE Disability-free life expectancy

HALE Health-adjusted life expectancy

Disability and Health 


$\begin{array}{ll}\text { LE } & \text { Life expectancy } \\ \text { LED } & \text { Life expectancy with disability } \\ \text { NDSS } & \text { National Diabetes Services Scheme } \\ \text { NDI } & \text { National Death Index } \\ \text { SDAC } & \text { Survey of Disability, Ageing and Carers } \\ \text { UI } & \text { Uncertainty interval }\end{array}$

\section{Introduction}

Diabetes is a major health problem worldwide, affecting 415 million people globally in 2015 [1]. In Australia, the prevalence of diabetes has more than doubled over the past three decades [2], with an estimate of around one million people living with diabetes in 2011-2012 and a large proportion of this burden being experienced by older people [3, 4]. It is predicted that more than one-third of today's young adults will develop diabetes during their lifetime and that $14 \%$ of their remaining life will be spent living with diabetes [5]. Meanwhile, owing to advances in treatment and better availability of diabetes care, mortality has declined $[6,7]$ and life expectancy (LE) [8] has increased for people with diabetes. A study from the USA reported that reduction in LE due to diabetes when diagnosed at age 40 years decreased from 7.7 years in 1990-1999 to 5.8 years in 2000-2011 in men and from 8.7 years to 6.8 years in women [8]. The rising prevalence, together with the improvement in longevity, has prompted a greater emphasis to be placed on the health-related quality of life in people with diabetes.

Diabetes and its associated complications and comorbidities have been suggested to lead to poor health and to physical disability among older adults [9-11], and impose a substantial burden on healthcare expenditure $[12,13]$. In a recent comprehensive meta-analysis of 26 studies [14], Wong et al found that there was a $50-80 \%$ increase in the odds of disability in diabetes compared with no diabetes.

There are two major classes of summary measures of population health: positive measures of health expectancy, such as health-adjusted life expectancy (HALE) and disability-free life expectancy (DFLE), and measures of health gaps such as disability-adjusted life-years (DALYs). DALYs are more commonly used to compare the burden of different diseases, often across different countries. DFLE, which is a focus of this study, incorporates both disability prevalence and mortality levels and has been widely employed to measure burdens of diseases. However, very few studies have provided estimates of the impact of diabetes on DFLE. In this study, we aimed to examine the contemporary burden of diabetes using data from the 2012 Survey of Disability, Ageing and Carers (SDAC) and national mortality data. We estimated LE and DFLE for individuals with and without diabetes and the gain in LE and DFLE after hypothetical elimination of diagnosed diabetes using a disease-delete method [15].

\section{Methods}

LE at an exact age is the average remaining years that will be lived by a group of individuals after that age, assuming agespecific mortality rates remain stable over time. LE is composed of lengths of time spent in different states of health until death, while DFLE is the number of remaining years, at a particular age, which an individual can expect to live in a disability-free state. DFLE is a composite indicator that combines morbidity and mortality into a single statistic. To calculate DFLE, age-specific mortality data taken from period life tables and data on the prevalence of disability are required.

Data sources Diabetes prevalence was derived from the Australian Diabetes, Obesity and Lifestyle (AusDiab) study, the methods of which have been described previously [16, 17]. In brief, the AusDiab study is the largest Australian longitudinal population-based study to examine the natural history of diabetes and heart and kidney diseases and has provided nationally representative data on the prevalence of diabetes in Australia.

All-cause mortality rates of people with diabetes in 2011 were determined through a linkage of the National Diabetes Services Scheme (NDSS) to the National Death Index (NDI). The NDSS was established by the Australian government in 1987 to deliver diabetes-related products at subsidised prices and to provide information to people with diabetes. Registration of patients is free and is completed by a medical practitioner or accredited diabetes educator. The NDSS captures $80-90 \%$ of all Australians with known diabetes [18]. The linkage method has been reported previously [7, 19]. National mortality rates for the general population in 2011 were provided by the Australian Institute of Health and Welfare (AIHW). To calculate mortality rates for the population without diabetes $\left(\mathrm{M}_{\mathrm{NDM}}\right)$, we used the following formula: $\mathrm{M}=\left[\mathrm{M}_{\mathrm{NDM}} \times(1-\mathrm{p})\right]+\left[\mathrm{M}_{\mathrm{DM}} \times \mathrm{p}\right]$, where $\mathrm{M}$ is the agespecific mortality rate for the total population, $\mathrm{M}_{\mathrm{DM}}$ is the age-specific mortality rate for the population with diabetes and $p$ is the age-specific diabetes prevalence rate derived from the AusDiab study.

The 2012 SDAC is the seventh comprehensive national survey conducted by the Australian Bureau of Statistics (ABS) to measure disability. Multi-stage sampling techniques were used to select the sample for the survey. After sample loss, the household sample included 27,410 private dwellings with a response rate of $89.8 \%$ and 518 non-private dwellings with a response rate of $80.4 \%$, while the cared-accommodation sample included 1,142 health establishments with a response rate of $87.5 \%$ [20]. The survey data are weighted by the ABS to address the issue of unequal probability of selection in the survey and to make the survey data a true representation of the Australian population. The 2012 SDAC provided information on the prevalence of disability by sex and 5 year age group in the general population. 
The age-specific disability prevalence in people with and without diabetes was derived by combining age-specific national disability prevalence from the 2012 SDAC, age-specific diabetes prevalence from the AusDiab study and RRs of disability associated with diabetes from Wong et al's meta-analysis [14]. The age-specific prevalence of disability in those with diabetes (Dd) and those without diabetes (Dnd) was then calculated using the following formulas: $\mathrm{D}=[\mathrm{Dnd} \times(1-\mathrm{p})]+[\mathrm{Dnd} \times \mathrm{RR} \times \mathrm{p}]$ and $\mathrm{Dd}=\mathrm{Dnd} \times \mathrm{RR}$, where $\mathrm{D}$ is the age-specific prevalence of disability for the total population, RR is the RR of disability in the population with diabetes relative to the population without diabetes and $p$ is the age-specific prevalence of diabetes.

Age was stratified into 5 year groups from age 50 up to age 79 years and an open-ended group thereafter. All data analyses were performed by each age group and sex.

Disability In the 2012 SDAC, the definitions of disability generally follow the conceptual framework of the International Classification of Functioning, Disability and Health (ICF). A person is defined as having a disability if they have at least one of 17 limitations, restrictions or impairments, which has lasted or is likely to last for at least 6 months and restricts everyday activities. People who were identified as having a disability were then asked about their need for assistance with the core activities of self-care (bathing or showering, dressing, eating, using the toilet and bladder or bowel control), mobility (getting into or out of a bed or chair, moving around at home and going to, or getting around, a place away from home) and communication (understanding and being understood by others: strangers, family and friends). Four levels of core activity limitation were determined, based on whether a person needs help, has difficulty or uses aids or equipment with any of the core activities. A person's overall level of core activity limitation was determined by the highest level of limitation in these core activities, the levels being classed as mild, moderate, severe and profound. For details of the survey's operational definitions of disability and severe or profound core activity limitation, please see the ABS website [20].

Statistical methods Abridged period life tables from age 50 years, which combined the age-specific mortality rates and age-specific disability prevalence, were constructed using Chiang's [21] and Sullivan's [22] method by 5 year age groups up to age 79 years and an open-ended group thereafter for each sex, conditional on surviving to the diagnosis date for the diabetes population. The life table output was LE, DFLE, life expectancy with disability (LED) and the years lived with severe or profound core activity limitation. Ninety-five per cent uncertainty intervals (UIs) for the life table measures were derived by analysing the impact of each of the key inputs using@ Risk Software (Version 7.0.0; Palisade, Ithaca, NY, USA). @ RISK is a software macro add-on for Microsoft
Excel that performs Monte Carlo simulation. With this method, inputs are entered as ranges rather than single values and each range is described by a probability distribution (trigen) to reflect the nature of uncertainty. In these analyses, the inputs for the @ RISK simulation were the point estimate and its $95 \%$ CIs. The CIs of these inputs were calculated using a 1,000 replicate bootstrap procedure. All estimates in our own work are given with $95 \%$ UI.

The gains in LE and DFLE for the whole population, after the hypothetical elimination of diagnosed diabetes, were calculated by the disease-deleted method [15]. According to this method the gain in LE (or DFLE) is the difference in LE (or DFLE) between the population without diabetes and the total population.

Sensitivity analyses were performed to investigate the effect of the RR of disability associated with diabetes on the estimates of DFLE, LED and the years lived with severe or profound core activity limitation. Wong et al's meta-analysis [14] reported that diabetes increased the risk of mobility disability ( 15 studies; OR 1.71, 95\% CI 1.53, 1.91; RR 1.51, 95\% CI $1.38,1.64)$, of instrumental activities of daily living disability (10 studies; OR $1.65,95 \%$ CI $1.55,1.74$ ) and of activities of daily living disability (ADLs) (16 studies; OR 1.82, 95\% CI 1.63, 2.04; RR 1.82, 95\% CI 1.40, 2.36). For the current study, the RR of disability associated with diabetes was taken to be 1.65 (95\% CI $1.55,1.74)$, which was the medium RR among the three RRs and also corresponded to the moderate degree of disability among the three disability definitions. Due to the lack of availability of age-specific estimates of the RR of diabetes-associated disability in the literature, we applied the same RR across all age groups. For the sensitivity analysis, the RRs of $1.51(1.38,1.64)$ and 1.82 $(1.40,2.36)$ were used for calculating disability prevalence in those with and without diabetes and then estimating the DFLE, LED and the years lived with severe or profound core activity limitation based on the abridged period life tables.

Data analysis was carried out using STATA version 12.1 (Statacorp, College Station, TX, USA), Microsoft Excel 2010 (Microsoft, USA) and @ RISK Version 7.0 (Palisade, Ithaca, NY, USA). This study was approved by the Alfred Hospital Health Human Ethics Committee and the AIHW Ethics Committee.

\section{Results}

Both the prevalence of all disability and the prevalence of severe or profound core activity limitation increased with age irrespective of diabetes status and sex and both were significantly higher in those with diabetes than in those without diabetes (Table 1). The estimated prevalence of all disability increased from $27.5 \%$ at age 50 years to $100 \%$ at age 80 years and over, for men with diabetes and from $26.3 \%$ to $100 \%$ for women with diabetes. The estimated prevalence of severe or 
Table 1 Age-specific prevalence of disability and severe or profound core activity limitation of adults in the 2012 SDAC, by diabetes status and sex

\begin{tabular}{|c|c|c|c|c|}
\hline \multirow[t]{2}{*}{ Age group, years } & \multicolumn{2}{|c|}{ People with diabetes } & \multicolumn{2}{|c|}{ People without diabetes } \\
\hline & All disability & S/PCA limitation & All disability & S/PCA limitation \\
\hline \multicolumn{5}{|l|}{ Men } \\
\hline $50-54$ & $27.5(24.4,30.8)$ & $5.7(4.7,6.8)$ & $15.9(14.5,17.4)$ & $3.5(2.9,4.1)$ \\
\hline $55-59$ & $40.1(35.2,45.3)$ & $8.3(6.6,10.2)$ & $20.4(18.3,22.9)$ & $5.1(4.1,6.2)$ \\
\hline $60-64$ & $47.0(39.6,56.2)$ & $11.6(9.4,14.4)$ & $28.9(25.0,33.6)$ & $7.1(5.8,8.7)$ \\
\hline $65-69$ & $59.4(51.5,68.3)$ & $14.4(11.7,17.5)$ & $35.5(31.3,40.7)$ & $8.8(7.1,10.8)$ \\
\hline $70-74$ & $61.1(53.2,71.4)$ & $16.9(13.6,20.9)$ & $38.2(33.3,43.8)$ & $10.3(8.4,12.5)$ \\
\hline $75-79$ & $81.2(68.8,98.1)$ & $21.9(17.6,27.3)$ & $48.4(41.4,57.9)$ & $13.3(10.8,16.4)$ \\
\hline $80+$ & $100(82.4,100)$ & $48.8(31.3,67.1)$ & $63.7(54.0,77.1)$ & $29.6(19.1,41.1)$ \\
\hline \multicolumn{5}{|l|}{ Women } \\
\hline $50-54$ & $26.3(23.7,29.3)$ & $8.4(7.1,9.7)$ & $16.0(14.6,17.5)$ & $5.1(4.3,5.9)$ \\
\hline $55-59$ & $34.8(30.9,39.1)$ & $10.4(8.5,12.7)$ & $21.2(19.2,23.6)$ & $6.3(5.3,7.4)$ \\
\hline $60-64$ & $50.3(44.9,56.4)$ & $14.5(12.1,17.1)$ & $30.6(27.6,33.8)$ & $8.8(7.4,10.2)$ \\
\hline $65-69$ & $59.5(53.7,66.0)$ & $13.8(11.3,16.3)$ & $36.1(33.2,39.3)$ & $8.4(7.0,9.9)$ \\
\hline $70-74$ & $65.9(58.3,74.5)$ & $18.9(15.6,22.4)$ & $40.0(36.1,44.5)$ & $11.5(9.5,13.4)$ \\
\hline $75-79$ & $78.5(67.4,93.0)$ & $30.1(24.3,37.5)$ & $47.7(41.1,55.9)$ & $18.3(15.1,22.1)$ \\
\hline $80+$ & $100(92.7,100)$ & $66.7(54.2,80.1)$ & $65.9(57.4,75.9)$ & $40.5(33.5,48.1)$ \\
\hline
\end{tabular}

Data are presented as mean prevalence (\%) with $95 \%$ UI in parentheses

S/PCA, severe or profound core activity profound core activity limitation increased from $5.7 \%$ at age 50 years to $48.8 \%$ at age 80 years and over, for men with diabetes, and from $8.4 \%$ to $66.7 \%$ for women with diabetes.

Table 2 shows the estimates of LE, DFLE, LED and the years lived with severe or profound core activity limitation. These estimates were based on a single summary estimate for RR of disability associated with diabetes of 1.65 (95\% CI $1.55,1.74)$ which was derived from Wong et al's metaanalysis [14]. The estimated LE and DFLE for people with diabetes were lower than for those without diabetes from age 50 years to age 80 years and over. Men with diabetes at age 50 years had an estimated LE of 30.2 (95\% UI 30.0, 30.4) years with $12.7(11.5,13.7)$ years of DFLE and another 17.5 $(16.4,18.7)$ years of LED, including $5.8(4.7,6.9)$ years lived with severe or profound core activity limitation. Compared with men without diabetes, men with diabetes at age 50 years had $3.2(3.0,3.4)$ years of estimated LE loss and 8.2 (6.7, 9.7) years of estimated DFLE loss. Women with diabetes at age 50 years could expect to have an estimated LE of 33.9 $(33.6,34.1)$ years with $13.1(12.3,13.9)$ years of DFLE and another $20.8(20.0,21.7)$ years of LED, including $9.2(8.1$, 10.4) years with severe or profound core activity limitation. In women, those with diabetes as compared with those without diabetes at age 50 years had $3.1(2.9,3.4)$ years of estimated LE loss and $9.1(7.9,10.4)$ years of estimated DFLE loss. The significant differences in estimates of LE and DFLE between those with and without diabetes could be observed across all age groups but gradually declined with increasing age.
The proportion of the estimated years lived with disability or severe or profound core activity limitation increased with age, irrespective of diabetes status and sex, but was much higher in people with diabetes than in those without diabetes and also higher in women than in men (Fig. 1). At age 50 years, the estimated years lived with disability accounted for $58.0 \%$ of total LE for men with diabetes and $61.4 \%$ of total LE for women with diabetes, compared with $37.5 \%$ and $40.0 \%$ of total LE, respectively, for men and women without diabetes (Fig. 1a). The proportion of the estimated years lived with severe or profound core activity limitation at age 50 years was $19.1 \%$ and $27.2 \%$, respectively, for men and women with diabetes and $12.2 \%$ and $17.2 \%$, respectively, for men and women without diabetes (Fig. 1b).

Overall, women had longer LE and LED than men, and the significant sex difference in estimated LE narrowed with age, while the sex difference in estimated LED plateaued between age 50 and 65 years, and then gradually reduced among the older population (Fig. 2). The pattern was similar in those with and without diabetes. However, the sex difference in estimated LED and in the estimated years lived with severe or profound core activity limitation for the population with diabetes was proportionally larger than that for the population without diabetes across all ages. There was no significant sex difference in estimated DFLE in those with or without diabetes.

The gain in LE at age 50 years for the whole population, after eliminating diagnosed diabetes was estimated to be 0.6 $(95 \%$ UI $0.5,0.6)$ years. The corresponding gain in DFLE was $1.8(1.0,2.8)$ years, including $1.1(0.3,1.8)$ years without severe or profound core activity limitation. 


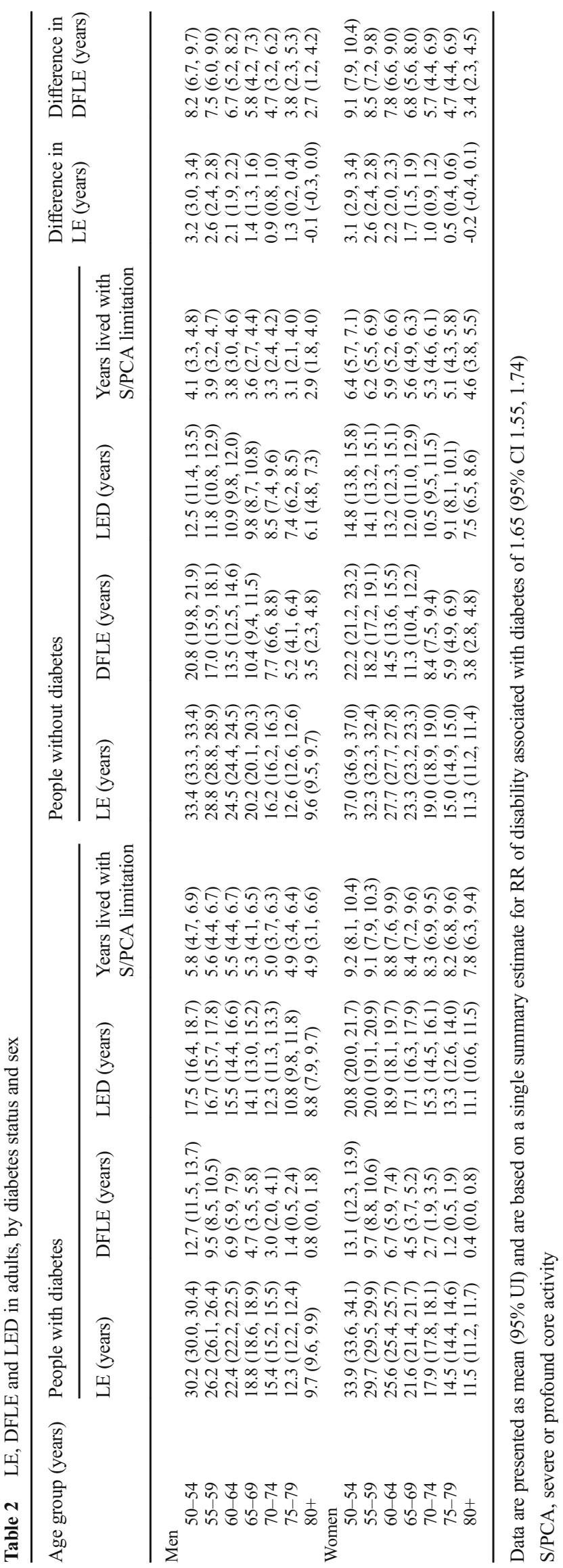



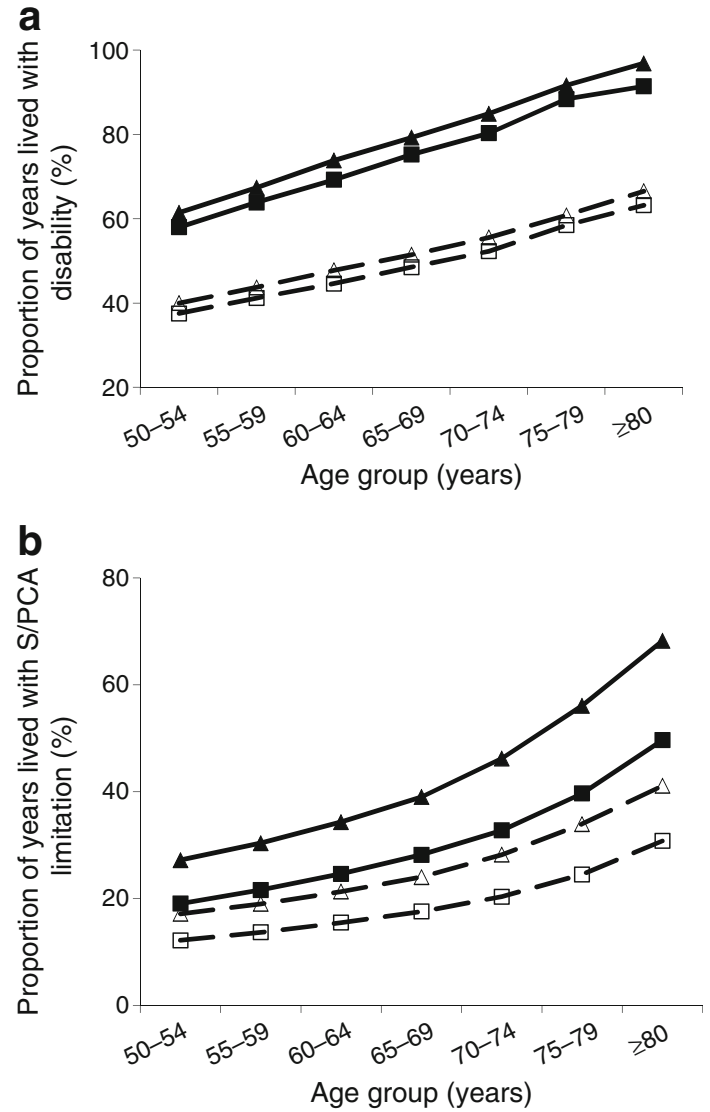

Fig. 1 Proportion of years lived with disability (a) and with severe or profound core activity (S/PCA) limitation (b), by sex. Solid line with black squares, men with diabetes; solid line with black triangles, women with diabetes; dashed line with white squares, men without diabetes; dashed line with white triangles, women without diabetes

In sensitivity analyses, we set the RR of disability associated with diabetes as $1.51(95 \% \mathrm{CI} 1.38,1.64)$ and $1.82(1.40$, 2.36), which were derived from Wong et al's meta-analysis [14] to calculate the disability prevalence in the population with and without diabetes and then constructed the life tables, separately (electronic supplementary material [ESM] Tables 1 and 2). The point estimates of DFLE for diabetes were a little higher $(\mathrm{RR}=1.51)$ or lower $(\mathrm{RR}=1.82)$ than the main study results but with overlapped UIs. For example, at age 50 years, the estimate of DFLE for men with diabetes was 16.9 (95\% UI $15.5,17.9)$ years when the RR was set as 1.51 and $14.5(12.3$, 16.4) years when the RR was set as 1.82 and was 15.9 (14.8, 16.9) years in the main study.

\section{Discussion}

In this study, we used a national survey of disability, a national cohort study of diabetes and national mortality data to estimate the disability burden of diabetes. The results showed that diabetes was associated with an estimated loss of LE and DFLE of 3.2 years and 8.2 years, respectively, for men and 3.1 years
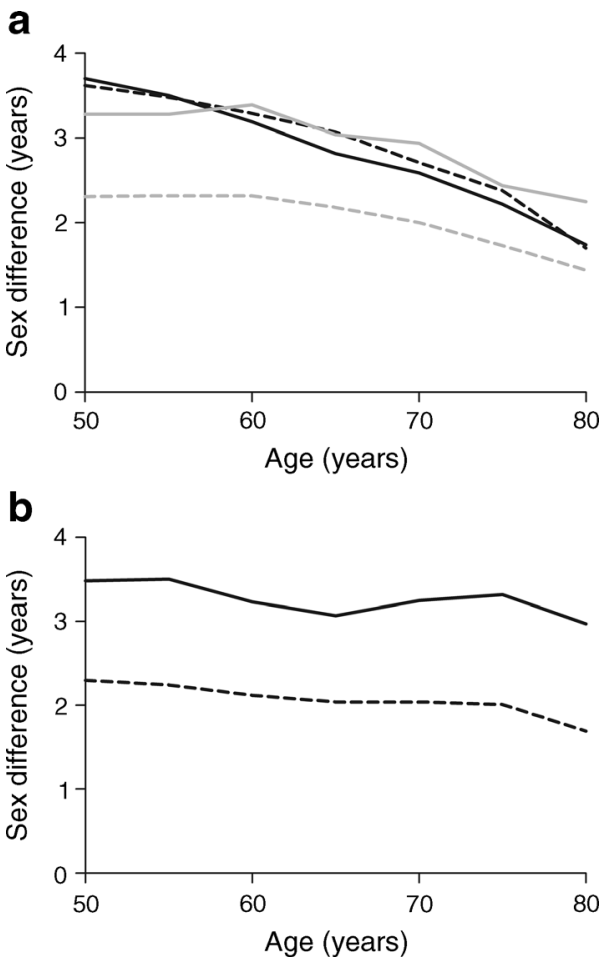

Fig. 2 Sex difference in LE and LED and the years lived with severe or profound core activity (S/PCA) limitation of adults, by diabetes status. Sex difference means positive values indicating greater LE (or LED, or the years lived with S/PCA limitation) in women than men. (a) Black solid line, LE in people with diabetes; black dashed line, LE in people without diabetes; grey solid line, LED in people with diabetes; grey dashed line, LED in people without diabetes. (b) Solid line, years lived with S/PCA limitation in people with diabetes; dashed line, years lived with S/PCA limitation in people without diabetes

and 9.1 years, respectively, for women, living with diabetes at age 50 years. Furthermore, women with diabetes spent more years and a greater proportion of life (61.4\% at age 50 years) with disability than did women without diabetes and men with diabetes.

LE and DFLE are two widely used measures with which to evaluate the impact of a disease. DFLE adds a quality dimension to LE. As expected, LE and DFLE for the population with diabetes were shorter than for the population without diabetes. Australian data from 10 years ago showed that the loss in LE was 5.6 years and 4.9 years for men and women with diabetes, respectively, from age 55 years [5], higher than the loss reported in the current study (corresponding estimates in the current study were 2.6 years for both sexes). LE for the population with diabetes has improved in the last two decades and the LE loss associated with diabetes has reduced. National population-based studies in the USA and Canada have demonstrated that diabetes has a substantial impact on the length and quality of life. In the USA, Narayan et al [23] reported LE and quality-adjusted life-years according to the age of diagnosis for the US population with diabetes in 2000. For example, among people diagnosed with diabetes at age 50 years, the 
loss in LE was 9.2 and 12.1 years for men and women, respectively, and the loss of quality-adjusted life-years was 14.5 and 18.0 years for men and women, all of which were much higher than the estimates in our study. One of the possible explanations for this difference could be that the study population in the USA comprised less contemporary cohorts, where follow-up occurred during time periods when mortality risk for diabetes may have been greater. Additionally, Narayan et al's study [23] calculated quality-adjusted life-years by weighting each year with diabetes by 0.75 of a year without diabetes, which is a less precise approach than ours. In Canada, using the Health Utilities Index to assess healthrelated quality of life, Manuel and Schultz [24] estimated that the LE of people with diabetes at age 55 years was, respectively, 6.4 and 8.2 years less than the LE for men and women without diabetes and that the HALE was 5.8 and 7.1 years less than that of men and women without diabetes in 1996-1997; Loukine et al [15] estimated 5.0 and 6.0 years of LE loss and 5.3 and 5.8 years of HALE loss associated with diabetes for men and women at age 55 years in 2000-2006. However, compared with the studies in Canada, our study reported a lower loss of LE (2.6 years for both sexes at age 55 years) but a larger loss of DFLE ( 7.5 years for women and 8.5 years for men at age 55 years) for the population with diabetes. Although results may not be directly comparable because there are differences in the methods used for measuring disability, the recent cohort and inclusion of the residents living in long-term care facilities in the 2012 SDAC might contribute to the difference between our study and the Canadian studies.

To further quantify the impact of diabetes on population health, we introduced the disease-deleted method and found that eliminating diagnosed diabetes would result in a compression of morbidity, as there would be only a small gain in LE ( 0.6 years at age 50 years) but a gain of nearly 2 years of DFLE. This suggests that the impact of diabetes on the quality of life is larger than the impact on length of life, which could partly be explained by an ageing population and declining mortality in diabetes [7].

A comparison between sexes revealed that women with diabetes lived longer than men with diabetes but spent a greater number of years and a greater proportion of their life with disability. The differential impact of diabetes by sex on LED can be explained largely by the fact that women with diabetes had longer LE and significantly higher prevalence of disability, which is consistent with previously reported data for women in general $[9,25]$.

The relationship between diabetes and disability is likely due to multiple factors. Many studies have shown that the greater burden of traditional diabetes complications and comorbidities [9, 11, 26, 27] and poor glycaemic control $[11,28]$ could contribute to the higher risk for disability associated with diabetes. Additionally, non-traditional diabetes-related problems, such as cognitive impairment and sarcopenia
[29-31], could also be the potential mechanisms that cause disability. The striking loss of DFLE in diabetes reported in this study is likely to raise concern about the burden of diabetes in future decades, indicating a need to respond by implementing intervention and prevention of disability. Evidence has shown that the loss of muscle mass and strength in diabetes may potentially be improved with insulin sensitisers [32]. In 2012, the Look AHEAD trial [33] found that intensive lifestyle intervention with a focus on weight loss and physical activity reduced the incidence of physical disability by half among overweight or obese adults with diabetes. Nonetheless, further research is still needed to explore other mechanisms (e.g. related to obesity or lack of physical activity) that lead to disability in diabetes. This will help inform strategies aimed at prevention and control of disability in those with diabetes.

When interpreting the results in this study, it is important to take into account that definitions of severity of disability may affect the estimates. A wide range of concepts and measures of disability has been used in the studies on population health. In the present study, the definition of disability was based on the conceptual framework of the ICF, which incorporates the medical and social aspects of people's health condition including all levels of disability. The definition of severe or profound core activities limitation is a combination of ADLs, mobility and communication, including severe and above levels of disability.

There are several advantages of combining data from a survey of national disability prevalence with a large national registry of diabetes. The 2012 SDAC was conducted at the national level using standard definitions of disability, following the ICF, thus could provide reliable prevalence estimates of disability. In addition, the AusDiab study that used an oral glucose tolerance test to define diabetes provided more definitive data on the true prevalence of diabetes. Since we linked the diabetes registry to the death index, we identified all deaths in people with diabetes, not just those identified on death certificates, which would be an underestimate [34].

There are several limitations that also must be acknowledged. First, our findings on the impact on disability are based on estimates, rather than on direct measurements, and the accuracy of these estimates depends on the quality of data input (e.g. the validity of the RRs for disability associated with diabetes). The RRs for disability associated with diabetes varied with the definition of severity of disability and none of the three definitions of disability in Wong et al's meta-analysis [14] is consistent with the ICF definition that we used in the current study. We chose the medium RR [1.65 (95\% CI 1.55, 1.74)], which corresponded to the moderate degree of disability among the three RRs in the major analysis. This could introduce bias in the estimates of DFLE, LED and the years lived with severe or profound core activity limitation. Although in the sensitivity analyses we found that a higher RR of 1.82 (95\% CI 1.40, 2.36) or lower RR of $1.51(1.38$, 
1.64) had negligible impact on the results, the potential bias due to not adjusting RRs for different ages is still present. Second, type 1 and type 2 diabetes were not distinguished in this study due to limitations in the data. Given the majority of diabetes in Australia is type 2 [35], the results in this study predominantly pertain to type 2 diabetes. The onset of type 1 diabetes is earlier than that of type 2 diabetes, which means a greater burden of complications and probably a higher risk of disability. Third, although the NDSS captures $80-90 \%$ of all Australians with known diabetes, it does not include those with undiagnosed diabetes and it may underrepresent those with diet-controlled diabetes. The mortality rate in the population with diabetes in this study might be overestimated, possibly leading to an underestimation of LE and DFLE for people with diabetes. Finally, LE and DFLE were estimated for people with and without diabetes using a period life table method. Period life table estimates are summary descriptive measures created from crosssectional data of open populations based on the past experience of the population. Therefore, estimates in this study should not be used to 'predict' LE, DFLE or LED for individuals, as the mortality and disability experience will change with time.

In summary, this study suggests that the LE of people with diabetes has improved but adults with diabetes are still afflicted with a considerable loss of DFLE. Efforts to identify the specific causes of disability and effective interventions are needed.

Acknowledgements We thank the NDSS, an initiative of the Australian government administered by Diabetes Australia since 1987, for the data source and thank the AIHW for the linkage of the NDSS to the NDI. The interpretation and conclusions contained in this study are those of the authors alone.

Funding This study was funded by the National Health and Medical Research Council (NHMRC Grant APP 1002663) and the Australian Research Council (Discovery Grant 120103277). This study is also supported in part by the Victorian Operational Infrastructure Program scheme. LH is supported by the China Scholarship Council. JES is supported by an NHMRC Senior Research Fellowship. EW and JLH are each supported by a Baker IDI Bright Sparks Scholarship and a Monash University Australian Postgraduate Award. AP is supported by an NHMRC Career Development Award.

Duality of interest The authors declare that there is no duality of interest associated with this manuscript.

Contribution statement LH performed the analysis and interpretation of data. JES made a substantial contribution to the analysis and interpretation of data. EW assisted in data analysis. JLH assisted in data preparation and data analysis. AP conceived the study and made a substantial contribution to the analysis and interpretation of data. DJM conceived the study and made a substantial contribution to the interpretation of data. $\mathrm{LH}$ wrote the first draft of the manuscript and JES, EW, JLH, AP and DJM reviewed and revised the manuscript. All authors approved the final version of the article. AP and DJM are the guarantors of this work and, as such, had full access to all the data in the study and take responsibility for the integrity of the data and the accuracy of the data analysis.

\section{References}

1. International Diabetes Federation (2015) IDF Diabetes Atlas update poster, 7th edn. International Diabetes Federation, Brussels

2. Dunstan DW, Zimmet PZ, Welborn TA et al (2002) The rising prevalence of diabetes and impaired glucose tolerance: the Australian Diabetes, Obesity and Lifestyle Study. Diabetes Care 25:829-834

3. Cowie CC, Rust KF, Ford ES et al (2009) Full accounting of diabetes and pre-diabetes in the U.S. population in 1988-1994 and 2005-2006. Diabetes Care 32:287-294

4. Narayan KM, Boyle JP, Geiss LS, Saaddine JB, Thompson TJ (2006) Impact of recent increase in incidence on future diabetes burden: U.S., 2005-2050. Diabetes Care 29:2114-2116

5. Magliano DJ, Shaw JE, Shortreed SM et al (2008) Lifetime risk and projected population prevalence of diabetes. Diabetologia 51: 2179-2186

6. Gregg EW, Cheng YJ, Saydah S et al (2012) Trends in death rates among U.S. adults with and without diabetes between 1997 and 2006: findings from the National Health Interview Survey. Diabetes Care 35:1252-1257

7. Harding JL, Shaw JE, Peeters A, Guiver T, Davidson S, Magliano DJ (2014) Mortality trends among people with type 1 and type 2 diabetes in Australia: 1997-2010. Diabetes Care 37:2579-2586

8. Gregg EW, Zhuo X, Cheng YJ, Albright AL, Narayan KM, Thompson TJ (2014) Trends in lifetime risk and years of life lost due to diabetes in the USA, 1985-2011: a modelling study. Lancet Diabetes Endocrinol 2:867-874

9. Gregg EW, Beckles GL, Williamson DF et al (2000) Diabetes and physical disability among older U.S. adults. Diabetes Care 23: $1272-1277$

10. Gregg EW, Mangione CM, Cauley JA et al (2002) Diabetes and incidence of functional disability in older women. Diabetes Care 25:61-67

11. Kalyani RR, Saudek CD, Brancati FL, Selvin E (2010) Association of diabetes, comorbidities, and $\mathrm{A} 1 \mathrm{C}$ with functional disability in older adults: results from the National Health and Nutrition Examination Survey (NHANES), 1999-2006. Diabetes Care 33: $1055-1060$

12. American Diabetes A (2013) Economic costs of diabetes in the U.S. in 2012. Diabetes Care 36:1033-1046

13. Leung MY, Pollack LM, Colditz GA, Chang SH (2015) Life years lost and lifetime health care expenditures associated with diabetes in the U.S., National Health Interview Survey, 1997-2000. Diabetes Care 38:460-468

14. Wong E, Backholer K, Gearon E et al (2013) Diabetes and risk of physical disability in adults: a systematic review and meta-analysis. Lancet Diabetes Endocrinol 1:106-114

15. Loukine L, Waters C, Choi BC, Ellison J (2012) Impact of diabetes mellitus on life expectancy and health-adjusted life expectancy in Canada. Popul Health Metrics 10:7

16. Dunstan DW, Zimmet PZ, Welborn TA et al (2002) The Australian Diabetes, Obesity and Lifestyle Study (AusDiab)-methods and response rates. Diabetes Res Clin Pract 57:119-129

17. Magliano DJ, Barr EL, Zimmet PZ et al (2008) Glucose indices, health behaviors, and incidence of diabetes in Australia: the Australian Diabetes, Obesity and Lifestyle Study. Diabetes Care $31: 267-272$

18. Australian Institute of Health and Welfare Diabetes (2009) Prevalence in Australia. An assessment of national data sources. Australian Institute of Health and Welfare, Canberra

19. Harding JL, Shaw JE, Peeters A, Cartensen B, Magliano DJ (2015) Cancer risk among people with type 1 and type 2 diabetes: disentangling true associations, detection bias, and reverse causation. Diabetes Care 38:264-270 
20. Australian Bureau of Statistics (2012) Disability, ageing and carers, Australia: summary of findings, 2012. Available from www.abs. gov.au/AUSSTATS/abs@.nsf/Lookup/4430.0Main+ Features12012?OpenDocument. Accessed 13 Oct 2016 (archived)

21. Chiang CL (1984) The life table and its construction - the abridged life table. In: Chiang CL (ed) The life table and its applications. Robert E. Krieger, Malabar, pp 137-152

22. Sullivan DF (1971) A single index of mortality and morbidity. HSMHA Health Rep 86:347-354

23. Narayan KM, Boyle JP, Thompson TJ, Sorensen SW, Williamson DF (2003) Lifetime risk for diabetes mellitus in the United States. JAMA 290:1884-1890

24. Manuel DG, Schultz SE (2004) Health-related quality of life and health-adjusted life expectancy of people with diabetes in Ontario, Canada, 1996-1997. Diabetes Care 27:407-414

25. Maggi S, Noale M, Gallina P et al (2004) Physical disability among older Italians with diabetes. The ILSA study. Diabetologia 47: 1957-1962

26. Volpato S, Blaum C, Resnick H et al (2002) Comorbidities and impairments explaining the association between diabetes and lower extremity disability: The Women's Health and Aging Study. Diabetes Care 25:678-683

27. Sinclair AJ, Conroy SP, Bayer AJ (2008) Impact of diabetes on physical function in older people. Diabetes Care 31:233-235

28. Bossoni S, Mazziotti G, Gazzaruso C et al (2008) Relationship between instrumental activities of daily living and blood glucose control in elderly subjects with type 2 diabetes. Age Ageing 37: 222-225

29. Kalyani RR, Corriere M, Ferrucci L (2014) Age-related and disease-related muscle loss: the effect of diabetes, obesity, and other diseases. Lancet Diabetes Endocrinol 2:819-829

30. Park SW, Goodpaster BH, Lee JS et al (2009) Excessive loss of skeletal muscle mass in older adults with type 2 diabetes. Diabetes Care 32:1993-1997

31. Park SW, Goodpaster BH, Strotmeyer ES et al (2007) Accelerated loss of skeletal muscle strength in older adults with type 2 diabetes: the health, aging, and body composition study. Diabetes Care 30: $1507-1512$

32. Lee CG, Boyko EJ, Barrett-Connor E et al (2011) Insulin sensitizers may attenuate lean mass loss in older men with diabetes. Diabetes Care 34:2381-2386

33. Rejeski WJ, Ip EH, Bertoni AG et al (2012) Lifestyle change and mobility in obese adults with type 2 diabetes. N Engl J Med 366: 1209-1217

34. Cheng WS, Wingard DL, Kritz-Silverstein D, Barrett-Connor E (2008) Sensitivity and specificity of death certificates for diabetes: as good as it gets? Diabetes Care 31:279-284

35. Australian Institute of Health and Welfare (2011) Diabetes prevalence in Australia: detailed estimates for 2007-08. Australian Institute of Health and Welfare, Canberra 